\title{
Using ceftazidime-avibactam for persistent carbapenem- resistant Serratia marcescens infection highlights antimicrobial stewardship challenges with new beta- lactam-inhibitor combination antibiotics
}

H D Tootla, ${ }^{1}$ MB ChB, FCPath (SA) Micro, MMed Path (Microbiology); J Copelyn, ${ }^{2}$ MBBS, FCPaed (SA), MMed (Paediatrics), MSc; A Botha, ${ }^{3}$ BPharm; A J Brink, ${ }^{1}$ MB ChB, MMed Path (Microbiology); B Eley, ${ }^{2}$ MB ChB, FCPaed (SA), BSc Hons

\author{
${ }^{1}$ Microbiology Laboratory, National Health Laboratory Service, Groote Schuur Hospital, Cape Town, and Division of Medical Microbiology, \\ Department of Pathology, Faculty of Health Sciences, University of Cape Town, South Africa \\ ${ }^{2}$ Paediatric Infectious Diseases Unit, Red Cross War Memorial Children's Hospital, and Department of Paediatrics and Child Health, Faculty of \\ Health Sciences, University of Cape Town, South Africa \\ ${ }^{3}$ Western Cape Department of Health, Red Cross War Memorial Children's Hospital Pharmacy, Cape Town, South Africa
}

Corresponding author: H D Tootla (hafsah.tootla@nhls.ac.za)

\begin{abstract}
The newer beta-lactam-inhibitor combination (BLIC) antibiotics are available in South Africa (SA) for the treatment of carbapenemresistant Enterobacterales infections. We describe the successful use of ceftazidime-avibactam (CA) for the treatment of a child with persistent carbapenem-resistant Serratia marcescens bacteraemia, and the challenges faced using this lifesaving antibiotic, including access to susceptibility testing, procurement process, cost and complexity of deciding when, how and for how long to use it. Furthermore, the burden of carbapenem resistance is increasing in SA, and inappropriate use of CA and other newer BLIC antibiotics, such as ceftolozanetazobactam, will inevitably endanger their longevity. A careful balance must be struck between removing unnecessary obstacles and delays in initiating these antibiotics for life-threatening infections, and additional antimicrobial stewardship-guided interventions aimed at preserving their therapeutic use.
\end{abstract}

S Afr Med J 2021;111(8):729-731. https://doi.org/10.7196/SAMJ.2021.v111i8.15762

Carbapenem-resistant Enterobacterales (CRE) is a global threat to human health. ${ }^{[1,2]}$ The main mechanism of resistance is production of carbapenemases, which hydrolyse most beta-lactam antibiotics. Clinical management is compounded by resistance conferred by plasmid-encoded genes to other classes of antibiotics, such as aminoglycosides, fluoroquinolones and colistin..$^{[1,2]}$ The newer beta-lactam-inhibitor combination (BLIC) antibiotics, such as ceftazidime-avibactam (CA), for serious CRE infections, are now available in South Africa (SA). However, these are not easily accessible and decisions regarding their appropriate use are complex. Furthermore, to ensure sustained effectiveness of these antibiotics, it is imperative that health systems guard against unnecessary use and recommit to infection prevention and control measures to prevent the spread of these organisms in hospitals. We describe a child with persistent carbapenem-resistant Serratia marcescens bacteraemia, who was successfully treated with CA. We also highlight some of the challenges faced using a new unregistered antibiotic (Table 1) and call for the development of antimicrobial stewardship (AMS)-guided 'best practices' for their use.

\section{Case presentation}

A 17-month-old previously well child was referred to our tertiary centre with extensive hot-water burns ( $46 \%$ total body surface area). After resuscitation she was admitted for wound debridement and dressings. During the first 2 weeks of admission, she was treated empirically for presumed sepsis multiple times. Four blood cultures, one catheter tip and two tracheal aspirate cultures did not yield growth of a bacterial pathogen. Antimicrobials used during this period included amoxicillin-clavulanic acid, clindamycin, piperacillintazobactam, amikacin, meropenem, vancomycin and fluconazole. Additional risk factors for CRE infection included placement of central venous pressure (CVP) catheters and mechanical ventilation during periods of clinical instability. ${ }^{[3]}$

On day 21 of admission, she developed a low-grade fever. Clinically, no source of infection was found, but procalcitonin increased from $0.3 \mu \mathrm{g} / \mathrm{L}$ to $3 \mu \mathrm{g} / \mathrm{L}$ over 72 hours. The child's CVP catheter was replaced and she was commenced empirically on meropenem, vancomycin and fluconazole. Culture from the CVP catheter tip and burn wound pus swab subsequently cultured CRE S. marcescens, and an extended-spectrum beta-lactamase (ESBL) producing $S$. marcescens, respectively. To reduce transmission of the CRE organism in the hospital, the patient was isolated and strict contact precautions were implemented. The ETEST (bioMérieux, France) determined CRE S. marcescens minimum inhibitory concentrations (MICs) of $>32 \mu \mathrm{g} / \mathrm{mL}$ for ertapenem, imipenem and meropenem. The presence of an oxacillinase- 48 (OXA-48) carbapenemase was phenotypically confirmed using the RESIST-4 OKNV kit (Coris Bioconcept, Belgium). As $S$. marcescens is inherently resistant to colistin, combination therapy with meropenem (40 mg/kg/dose 8-hourly) and amikacin was initiated, and after completion of 8 days of targeted therapy, a good clinical response was observed. 
Table 1. Some challenges faced with use of ceftazidime-avibactam for carbapenem-resistant Enterobacterales infection

\author{
Administrative challenges leading to delays in early administration \\ Cost \\ Section 21 drug - requires consent and approval from local drug and therapeutics committees and SAHPRA \\ Diagnostic challenges leading to delays in early administration \\ Culture-based diagnostics are slow (24 - 72 hours) \\ Characterisation of the type of carbapenemase produced is required \\ Susceptibility testing of newer BLICs is not available in public sector laboratories and adds 24 - 48 hours to routine culture-based diagnostics \\ Clinical management challenges \\ Lack of AMS-guided 'best practices' for their use, describing when, how and for how long to use the newer BLICs \\ Uncertainty regarding the use of BLICs as monotherapy or combination therapy with other antibiotics
}

SAHPRA = South African Health Products Regulatory Authority; BLIC = beta-lactam-inhibitor combination; AMS = antimicrobial stewardship

However, 3 days later the patient deteriorated and required mechanical ventilation and inotropes. Meropenem, amikacin, tigecycline, vancomycin and fluconazole were commenced empirically. CRE S. marcescens and carbapenem-resistant Acinetobacter baumannii (CRAB) were isolated from blood culture and wound pus swabs. Vancomycin and fluconazole were discontinued, and colistin was used to treat CRAB. During the next month, her clinical course was unsteady, fluctuating between improvement and rapid deterioration, requiring inotropic and ventilatory support. Exhaustive investigation, including 2 transoesophageal echocardiograms, a bone scan and multiple wound inspections and dressing changes in theatre, did not reveal the source of the infection. CRE S. marcescens was persistently isolated from serial blood cultures performed every $24-48$ hours, despite continuous antimicrobial therapy that was selected based on susceptibility test results of cultured organisms. Antimicrobial choices included combinations with meropenem, amikacin, tigecycline, trimethoprim-sulfamethoxazole (TS) and colistin.

As there was persistent bacteraemia and the patient's clinical condition was worsening, we requested susceptibility testing for CA from a private laboratory. After confirmation of susceptibility, we obtained parental consent, and applied for and received approval from the institution's drug and therapeutic committee and the South African Health Products Regulatory Authority (SAHPRA) to use CA, as it is currently unregistered in SA.

At the time of CA initiation, the patient was critically unwell. She had generalised oedema, persistent thrombocytopenia, was mechanically ventilated and receiving inotropes, and intravenous access was difficult. Blood cultures at this time isolated a non-fermentative Gram-negative organism and CRE S. marcescens. CA was commenced $(50 \mathrm{mg} / \mathrm{kg} /$ dose 8-hourly) in combination with tigecycline, amikacin and colistin. Colistin was discontinued once the non-fermentative Gram-negative organism was identified as a wild-type Pseudomonas aeruginosa. After 7 days, the patient had improved and was moved to the ward for wound care. Unfortunately, 72 hours later, she developed a fever and increasing respiratory distress, requiring readmission to the intensive care unit (ICU) for mechanical ventilation and inotropes. CRE S. marcescens organisms were again isolated from blood cultures, and this relapse was suspected to have been due to inadvertent administration of ceftazidime instead of CA. Once the blood culture result became available, CA was used in combination with TS and amikacin, based on the antibiotic susceptibilities of the most recent CRE S. marcescens. Amikacin was stopped after clinical stability was achieved and repeat blood cultures were negative, because of concerns over prolonged aminoglycoside exposure and its associated toxicity. She completed a further 4 weeks of CA and TS, with good clinical response. After 116 days in hospital, the patient was discharged home, with follow-up appointments for wound care and skin grafts.

\section{Discussion}

Despite calls for awareness and prevention strategies being implemented to prevent the spread of multidrug-resistant organisms in health systems, the prevalence of CRE in SA is increasing. ${ }^{[4,5]}$ Mortality due to infection caused by these organisms is high and therapeutic options for treatment are limited. Guidelines detailing optimal management of such infections are not available and evidence-based data are emerging, making treatment decisions complicated, as seen in our patient. . $^{[1,25]}$

Our patient was managed by a multidisciplinary team comprising microbiologists, infectious disease sub-specialists, pharmacists, nursing staff and the treating clinicians. An individualised treatment regimen was administered based on the organisms isolated, availability of few susceptible antibiotics and use of last-line antibiotics. The CRE S. marcescens had consistently high MICs $(>32 \mu \mathrm{g} / \mathrm{mL})$ to the carbapenems, and although susceptible to amikacin, MICs were at the upper limit of susceptibility $(\leq 16 \mu \mathrm{g} / \mathrm{mL}){ }^{[6]}$ Based on this and poor clinical status, we initially treated our patient with meropenem, amikacin and tigecycline. MICs to tigecycline were low $(1 \mu \mathrm{g} / \mathrm{mL})$ and because no other routinely available antimicrobial was susceptible, the agent was used, despite no established interpretive criteria available to guide therapy for infection with $S$. marcescens. ${ }^{[6]}$ During the course of infection, tigecycline was replaced with TS, as later isolates were susceptible. When faced with clinical failure and persistent bacteraemia despite treatment, we investigated using CA as a last attempt to cure our patient, as in vitro activity and good clinical outcomes against class D carbapenemase OXA-48 have been described. ${ }^{[3,7,8]}$

CA is a new BLIC antibiotic effective against specific carbapenemases, i.e. OXA-48 and Klebsiella pneumoniae carbapenemase (KPC). ${ }^{[3,9]}$ It is currently available as a section 21 drug and is costly in SA. While characterisation of the type of carbapenemase produced by CRE is routinely provided by our public laboratory, susceptibility testing for CA is not offered by us or routinely offered by other public laboratories. Confirming susceptibility to CA at a private laboratory added $24-48$ hours to routine culture-based diagnostics. As CA is unregistered in SA, it was not available on site and required parental consent and approvals from local drug and therapeutic committees, as well as SAHPRA, before an order could be placed with the importing company. This process can vary from $\sim 7$ days to 6 weeks, depending on the turnaround time for the listed approvals and availability of local stock, hindering fast access to a lifesaving antibiotic.

In our patient, approval and procurement of a 7-day supply of stock occurred within 1 week, and we were able to commence our patient on CA. However, at the time, the importing company was facing a nationwide shortage of CA. Demand for the limited 
available stock of the medication was high and securing further supply for our patient was time-consuming and required numerous communications with the importing company. Determining how much stock to have on hand, by balancing cost and potential wastage, was an additional challenge due to the persistent bacteraemia and lack of an identifiable source. The package insert received with procured stock was in German. However, we obtained an English version from the manufacturer's website. It was used to train nursing staff to mix and administer the drug. In this regard, a further challenge was staff unfamiliarity with the drug. Ceftazidime is an antimicrobial that is used routinely in our institution and we suspect that ceftazidime, instead of CA, may have inadvertently been administered during the treatment course, with subsequent deterioration of our patient.

Burns patients are prone to persistent invasive infections and consequently exposed to prolonged courses of multiple antimicrobial agents, often with overlapping toxicity profiles. Inability to identify and control the source of infection, as with our patient, further extends treatment duration and complexity. Due to concerns regarding development of resistance, lack of identifiable source and prolonged duration of blood culture positivity, we used CA in combination with amikacin and TS. Furthermore, despite $S$. marcescens being intrinsically resistant to colistin, it was often included in the empirical management of clinical deterioration because of prior cultures with CRAB. Whether CA should be used as monotherapy or in combination with other antibiotics, has yet to be determined; however, data suggest that monotherapy might be appropriate. ${ }^{[10]}$ Paediatric specific data, especially in children $<3$ months old with infections other than those of the abdominal and urinary tracts, are urgently required, especially in neonates, where antibiotic therapy is often extended to include 'meningitis cover. ${ }^{\left[{ }^{[3]}\right.}$ Finally, a pertinent question regarding AMS is whether the agent should have been used earlier in the course of management or as a last resort, potentially limiting overall antimicrobial exposure and morbidity.

While new antibiotics such as CA have been shown to be superior to aminoglycosides and colistin for certain CRE infections, ${ }^{[8]}$ the new BLICs approved in some countries, or in clinical development, with activity against multidrug-resistant Gram-negative bacteria, will only partially address the currently prevalent mechanisms of resistance and are dependent on accurate and prompt identification of the organism and susceptibility testing. The mechanisms by which Gram-negative bacteria develop and express resistance may interfere with several facets of AMS and clinical treatment pathways, including choice of empirical regimen, potential for de-escalation and management of clinical failure due to the emergence of resistance during therapy. Therefore, the introduction of these agents into clinical use requires development of AMS-guided 'best practices'.

\section{Conclusions}

Infection prevention and control measures remain one of the most important tools in reducing morbidity and mortality caused by drug-resistant organisms. However, the use of CA and other newer BLIC antibiotics can be lifesaving for individual patients. Our case highlights the multiple barriers to using these drugs, including cost and long procurement processes, as well as the complexities of deciding when, how and for how long to use them. As clinician awareness of the availability and effectiveness of these drugs increases, the demand for their use can be expected to grow. Uncontrolled and unsuitable use will inevitably endanger their longevity and must be guarded against. In this regard, additional AMS-guided interventions and positioning for their use are required nationally.

Patient consent. Consent was obtained from the patient's parents to write and publish this case report.

Declaration. None.

Acknowledgements. We would like to thank the staff at Red Cross War Memorial Children's Hospital and the Microbiology Laboratory, National Health Laboratory Service, Groote Schuur Hospital, who were involved in the management of this case.

Author contributions. HDT, JC and BE conceptualised the article. HDT and JC wrote the original draft. HDT, JC, AB, AJB and BE were involved with editing and reviewing the paper.

Funding. None.

Conflicts of interest. None.

1. Brink AJ. Epidemiology of carbapenem-resistant Gram-negative infections globally. Curr Opin Infect Dis 2019;32(6):609-616. https://doi.org/10.1097/QCO.0000000000000608

2. Aguilera-Alonso D, Escosa-Garcia L, Saavedra-Lozano J, Cercenado E, Baquero-Artigao F Carbapenem-resistant Gram-negative bacterial infections in children. Antimicrob Agents Chemother 2020;64(3). https://doi.org/10.1128/AAC.02183-19

3. Bassetti M, Peghin M, Mesini A, Castagnola E. Optimal management of complicated infections in the pediatric patient: The role and utility of ceftazidime/avibactam. Infect Drug Resist 2020;13:17631773. https://doi.org/10.2147/IDR.S209264

4. Brink A, Feldman C, Richards G, Moolman J, Senekal M. Emergence of extensive drug resistance (XDR) among Gram-negative bacilli in South Africa looms nearer. S Afr Med J 2008;98(8):586-590.

5. Perovic O, Ismail H, Quan V, et al. Carbapenem-resistant Enterobacteriaceae in patients with Perovic O, Ismail H, Quan V, et al. Carbapenem-resistant Enterobacteriaceae in patients with
bacteraemia at tertiary hospitals in South Africa, 2015 to 2018. Eur J Clin Microbiol Infect Dis bacteraemia at tertiary hospitals in South Africa, 2015 to 2018. Eu

6. Clinical and Laboratory Standards Institute. Performance Standards for Antimicrobial Susceptibility Testing. 30th ed. CLSI supplement M100. Wayne, PA: CLSI, 2020.

7. Iosifidis E, Chorafa E, Agakidou E, et al. Use of ceftazidime-avibactam for the treatment of extensively drug-resistant or pan drug-resistant Klebsiella pneumoniae in neonates and children $<5$ years of age. Pediatr Infect Dis I 2019;38(8):812-815. https://doi.org/10.1097/INF.0000000000002344

8. Shields RK, Nguyen MH, Chen L, et al. Ceftazidime-avibactam is superior to other treatment

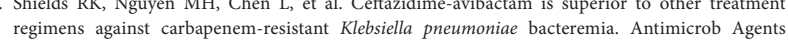
regimens against carbapenem-resistant Klebsiella pneumonia
Chemother 2017;61(8). https://doi.org/10.1128/AAC.00883-17

Chemother 2017;61(8). https://doi.org/10.1128/AAC.00883-17
9. Paterson DL, Isler B, Stewart A. New treatment options for multiresistant Gram negatives. Curr Opin Infect Dis 2020;33(2):214-223. https://doi.org/10.1097/QCO.0000000000000627

10. Fiore M, Alfieri A, Di Franco S, et al. Ceftazidime-avibactam combination therapy compared to ceftazidime-avibactam monotherapy for the treatment of severe infections due to carbapenemresistant pathogens: A systematic review and network meta-analysis. Antibiotics 2020;9(7). https: $/$ doi.org/10.3390/antibiotics 9070388 